\title{
Automatic calculation of one-loop amplitudes
}

\section{Andreas van Hameren*}

The H. Niewodniczański Institute of Nuclear Physics

Polisch Academy of Sciences

Radzikowskiego 152, 31-342 Krakow, Poland

E-mail: hameren@ifj.edu.pl

\section{C.G. Papadopoulos}

Institute of Nuclear Physics, NCSR Demokritos, 15310 Athens, Greece

E-mail: costas.papadopoulos@cern.ch

\section{R. Pittau}

Departamento de Física Teórica y del Cosmos

Centro Andaluz de Física de Partículas Elementales (CAFPE)

Universidad de Granada, E-18071 Granada, Spain

E-mail: pittau@ugr.es

An algorithm is presented to automatically compute any one-loop amplitude. It is an implementation involving the program CUTTOOLS for OPP-reduction, HELAC for the evaluation of tree-level off-shell currents and ONELOOP for the evaluation of one-loop scalar integrals. It is able to deal with at least all sub-processes included in the 2007 Les Houches wish list for LHC involving 3 or 4 particles in the final state, and has proven its usefulness in full NLO calculations.

RADCOR 2009 - 9th International Symposium on Radiative Corrections (Applications of Quantum Field Theory to Phenomenology)

October 25-30 2009

Ascona, Switzerland

This work was partially supported by RTN European Programme, MRTN-CT-2006-035505 (HEPTOOLS, Tools and Precision Calculations for Physics Discoveries at Colliders).

* Speaker. 


\section{Introduction}

In order to deal with the data from the experiments at LHC for the study of elementary particles, signals and potential backgrounds for new physics have to be under control at sufficient accuracy [1]. In particular, hard processes with high multiplicities, involving many particles or partons, cannot be neglected. Furthermore, such processes have to be dealt with at the next-to-leading order (NLO) level to, for example, reduce the scale dependence of observables and to have a better description of the shape of their distributions.

At the leading order in perturbation theory, many tools are already available that are able to deal with any scattering process involving up to several partons [2]. These tools are highly automated and they have been widely used [3]. At NLO the situation is less advanced, but there has been considerable progress recently. MCFM [4] is able to produce results at NLO accuracy for specific scattering processes, based on analytic calculations. Regarding the calculation of one-loop amplitudes, the only automatic tool available for some time now was FEYNCALC [5] and ForMCALC [6]. These tools rely heavily on the use of computer algebra. For processes with two particles in the final state, their performance is very satisfactory. There exist several important calculations that make use of these automatic packages and FEYNARTS [7], QGRAF [8], producing results with up to four particles in the final state [9], but for the moment no publicly available automatic tool exists. Recently a program called GOLEM [10] has been presented, that is able to deal with processes up to six external legs [11].

The aforementioned programs express one-loop amplitudes in Feynman graphs, which again are expressed in terms of one-loop tensor integrals. These are then calculated using universal reduction techniques, independent of the amplitude, by expressing them in terms of scalar integrals. Whereas this so-called tensor reduction happens usually numerically, the expression in terms of tensor integrals is obtained algebraically and turned into a computer program for numerical evaluation. These expressions can be huge, and a fully numerical approach could overcome this obstacle to large multiplicities.

One possibility is to still make use of tensor integrals, and calculate the tensors they are eventually contracted with numerically using recursive Dyson-Schwinger equations [12]. A very different line of thinking is known under the name of unitarity approach was set forward starting from the pioneering work in [13]. It proved to be very powerful in computing multi-parton amplitudes in QCD that seemed to be impossible with the traditional Feynman graph approach. The reason is that one-loop amplitudes are calculated by using tree-order building blocks, that are either known analytically with very compact expressions, or can be evaluated using fast recursive equations. Using the crucial input from [14], a fully numerical approach was presented in [15], introducing a systematic framework in order to calculate all coefficients of the scalar integrals, as well as part of the so-called rational contribution, related to the occurrence of UV-divergences. The remaining rational part can be reproduced by counter terms encoded in tree-like Feynman rules involving up to four fields [16]. Therefore, this method, known as the OPP method, provides a self-contained framework for the evaluation of the full one-loop amplitude. In [17], the OPP method was applied within the so-called generalized unitarity approach $[14,18]$ in order to get also the full rational contribution to the amplitude, paying the price to work with tree-amplitudes in higher dimensions.

The systematic extraction of all coefficients and of the rational term opened the road for the 
construction of tools that are able to compute one-loop amplitudes with any number of particles. BLACKHAT [19] and RocKet [20] were the first tools to realize such a possibility. In the following, we report on the development of another implementation.

\section{The program}

For any one-loop amplitude, a number $n$ of propagator denominators can be identified such that the amplitude can be represented in the form

$$
\mathscr{A}^{(1)}=\sum_{I \subset\{1, \ldots, n\}} \int \frac{\mu^{4-d} d^{d} \bar{q}}{(2 \pi)^{d}} \frac{\bar{N}_{I}(\bar{q})}{\prod_{i \in I} \bar{D}_{i}(\bar{q})} \quad, \quad \bar{D}_{i}(\bar{q})=\left(\bar{q}+p_{i}\right)^{2}-m_{i}^{2},
$$

where the loop momentum $\bar{q}$ and the numerator polynomials $\bar{N}_{I}(\bar{q})$ are considered to be evaluated in $d$ dimensions. The $p_{i}$ are combinations of the external momenta, and the $m_{i}$ are the masses of particles running a loop. It is well-known that for $d \rightarrow 4$, each such term can be cast into the form

$$
\int \frac{\mu^{4-d} d^{d} \bar{q}}{(2 \pi)^{d}} \frac{\bar{N}_{I}(\bar{q})}{\prod_{i \in I} \bar{D}_{i}(\bar{q})}=\sum_{j} d_{j} \operatorname{Box}_{j}+\sum_{j} c_{j} \operatorname{Tri}_{j}+\sum_{j} b_{j} \operatorname{Bub}_{j}+\sum_{j} a_{j} \operatorname{Tad}_{j}+R
$$

where Box, Tri, Bub and Tad refer to the one-loop scalar integrals with four, three, two and one external leg, and $R=R_{1}+R_{2}$ is the rational term. Given the momenta $p_{i}$, the masses $m_{i}$ and a function evaluating the 4-dimensional numerator $N_{I}$ as function of $q$, the program CUTTOOLS [21] identifies the scalar integrals to be evaluated, calculates the coefficients, and determines $R_{1}$ following the OPP method. The scalar integrals can be evaluated with the tools from [22] or with our own code ONELOOP.

For the evaluation of the numerator, the program HELAC [23] is used. It efficiently calculates tree-level amplitudes by applying recursive relations for off-shell currents [24]. The convenience of its applicability stems from the freedom in the choice of the initial decomposition represented by the summation in Eq. (2.1). In our set-up, it is a sum over all topologically inequivalent partitions of the external particles. For a one-loop amplitude with six external legs, for example, we have

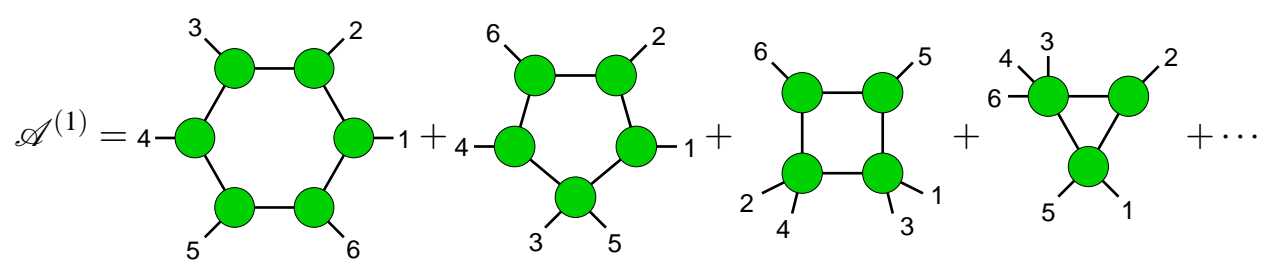

Each graph in the formula above corresponds to an instance of the label $I$ in Eq. (2.1). The blobs do not contain propagators depending on the integration momentum $q$. HeLAC can easily evaluate such a term by considering one line to be cut, and summing over all possible internal spin, flavor and/or color states of that cut line. On the right, for example, the second graph above is cut. Each term in this sum is an amplitude with two more external particles, restricted such that it only contains contributions of Feynman graphs including the propagators eventually forming the loop. This can easily be accomplished by putting together the necessary off-shell currents provided by HELAC.

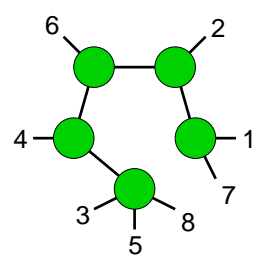


Regarding the color-treatment, HELAC uses the color-connection representation. External quarks and anti-quarks are represented by a color or anti-color index, gluons are represented by a pair of color/anti-color indices, and HELAC calculates all non-zero tree-level color-connections. The same is done to for the amplitudes with two more particles necessary to calculate the one-loop numerators. The contributions from non-planar loops are obtained by taking into account different orderings of these extra particles among the other external particles. For example, whereas

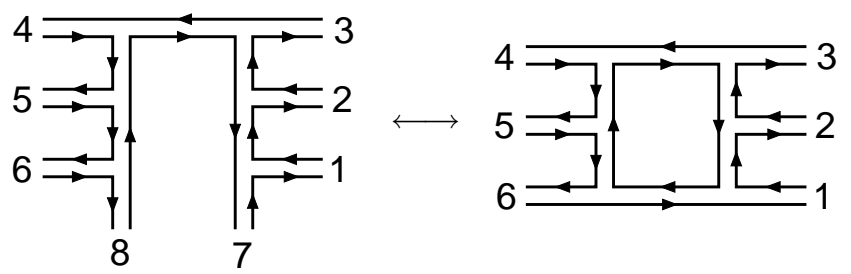

gives a planar contribution, exchanging 8 with 5 gives a non-planar contribution

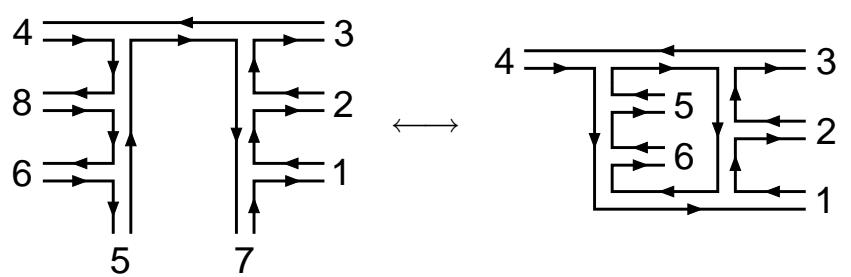

The evaluation of the rational part $R_{2}$, finally, follows the same line as the calculation of the counter terms necessary for renormalization, and is comparably trivial.

\section{Summary}

We presented a program to evaluate any one-loop amplitude, involving any Standard Model particles, for any momentum, color, and helicity configuration of the external particles. The calculation is performed in an automatic way, and is purely numerical. It is able to deal with at least all sub-processes included in the 2007 Les Houches wish list for LHC involving 3 or 4 particles in the final state. In combination with the implementation HelaC-DipOles [25] of the dipole-subtraction method, it has been used, and has proven its usefulness, in differential full NLO calculations [26].

\section{References}

[1] Z. Bern et al. [NLO Multileg Working Group], arXiv:0803.0494 [hep-ph].

[2] M. L. Mangano, M. Moretti, F. Piccinini, R. Pittau and A. D. Polosa, JHEP 0307 (2003) 001.

F. Maltoni and T. Stelzer, JHEP 0302 (2003) 027.

T. Gleisberg, S. Hoche, F. Krauss, A. Schalicke, S. Schumann, J. C. Winter, JHEP 0402 (2004) 056.

A. Kanaki and C. G. Papadopoulos, arXiv:hep-ph/0012004.

C. G. Papadopoulos, Comput. Phys. Commun. 137 (2001) 247.

A. Cafarella, C. G. Papadopoulos and M. Worek, arXiv:0710.2427 [hep-ph].

W. Kilian, T. Ohl and J. Reuter, arXiv:0708.4233 [hep-ph].

[3] J. Alwall et al., Eur. Phys. J. C 53 (2008) 473. 
[4] R. K. Ellis, Nucl. Phys. Proc. Suppl. 160 (2006) 170.

[5] R. Mertig, M. Bohm and A. Denner, Comput. Phys. Commun. 64 (1991) 345.

[6] T. Hahn, arXiv:0901.1528 [hep-ph].

[7] T. Hahn, Comput. Phys. Commun. 140 (2001) 418.

[8] P. Nogueira, J. Comput. Phys. 105 (1993) 279.

[9] W. Beenakker, S. Dittmaier, M. Kramer, B. Plumper, M. Spira, P. Zerwas, Nucl.Phys.B653(2003)151.

A. Denner, S. Dittmaier, M. Roth and L. H. Wieders, Phys. Lett. B 612 (2005) 223.

S. Dittmaier, P. Uwer and S. Weinzierl, Phys. Rev. Lett. 98 (2007) 262002.

S. Dittmaier, S. Kallweit and P. Uwer, Phys. Rev. Lett. 100 (2008) 062003.

A. Bredenstein, A. Denner, S. Dittmaier and S. Pozzorini, JHEP 0808 (2008) 108.

A. Bredenstein, A. Denner, S. Dittmaier and S. Pozzorini, Phys. Rev. Lett. 103 (2009) 012002.

[10] T. Binoth, J. P. Guillet, G. Heinrich, E. Pilon and T. Reiter, Comput. Phys. Commun. 180 (2009) 2317.

[11] T. Binoth, N. Greiner, A. Guffanti, J. P. Guillet, T. Reiter and J. Reuter, arXiv:0910.4379 [hep-ph].

[12] A. van Hameren, JHEP 0907, 088 (2009).

[13] Z. Bern, L. J. Dixon, D. C. Dunbar and D. A. Kosower, Nucl. Phys. B 435 (1995) 59.

Z. Bern, L. J. Dixon, D. C. Dunbar and D. A. Kosower, Nucl. Phys. B 425 (1994) 217.

[14] R. Britto, F. Cachazo and B. Feng, Nucl. Phys. B 725 (2005) 275.

[15] G. Ossola, C. G. Papadopoulos and R. Pittau, Nucl. Phys. B 763 (2007) 147.

G. Ossola, C. G. Papadopoulos and R. Pittau, JHEP 0707 (2007) 085.

[16] G. Ossola, C. G. Papadopoulos and R. Pittau, JHEP 0805 (2008) 004.

P. Draggiotis, M. V. Garzelli, C. G. Papadopoulos and R. Pittau, JHEP 0904 (2009) 072.

M. V. Garzelli, I. Malamos and R. Pittau, arXiv:0910.3130 [hep-ph].

[17] W. T. Giele, Z. Kunszt and K. Melnikov, JHEP 0804 (2008) 049.

R. K. Ellis, W. T. Giele, Z. Kunszt and K. Melnikov, arXiv:0806.3467 [hep-ph].

A. Lazopoulos, arXiv:0812.2998 [hep-ph].

[18] C. Anastasiou, R. Britto, B. Feng, Z. Kunszt and P. Mastrolia, Phys. Lett. B 645 (2007) 213.

[19] C. F. Berger et al., Phys. Rev. D 78 (2008) 036003.

[20] W. T. Giele and G. Zanderighi, JHEP 0806 (2008) 038.

[21] G. Ossola, C. G. Papadopoulos and R. Pittau, JHEP 0803 (2008) 042.

[22] G. J. van Oldenborgh, Comput. Phys. Commun. 66 (1991) 1.

R. K. Ellis and G. Zanderighi, JHEP 0802 (2008) 002.

[23] A. Kanaki and C. G. Papadopoulos, Comput. Phys. Commun. 132 (2000) 306.

[24] F. A. Berends and W. T. Giele, Nucl. Phys. B 306 (1988) 759.

F. Caravaglios and M. Moretti, Phys. Lett. B 358 (1995) 332.

P. Draggiotis, R. H. P. Kleiss and C. G. Papadopoulos, Phys. Lett. B 439 (1998) 157.

[25] M. Czakon, C. G. Papadopoulos and M. Worek, JHEP 0908 (2009) 085.

[26] G. Bevilacqua, M. Czakon, C. G. Papadopoulos, R. Pittau and M. Worek, JHEP 0909 (2009) 109. 